\title{
Challenges of Teaching Clinically Applied Anthropology and Cultural Psychiatry in India: An Evolving Partnership between a UK University and an Indian NGO
}

\author{
Jane Derges \\ University College London \\ Sumeet Jain \\ University College London and University of Edinburgh \\ Roland Littlewood \\ University College London \\ Vandana Gopikumar \\ ${ }^{2}$ The Banyan Academy of Leadership in Mental Health, Chennai \\ Mirjam Dijkxhoorn \\ ${ }^{2}$ The Banyan Academy of Leadership in Mental Health, Chennai \\ Sushrut Jadhav \\ University College London \\ Correspondence: s.jadhav@ucl.ac.uk
}

\begin{abstract}
The current emphasis on Global Mental Health risks losing the focus on the local and the particular and rendering anthropological insight pertinent. A more critical examination of pedagogical methods and curricula, and of the challenges of establishing collaborative, balanced partnerships is required.
\end{abstract}

Since 2006, a group of clinical anthropologists ${ }^{1}$ based at UCL and members of The Banyan ${ }^{2}$, have been working together on an innovative series of short, annual courses on social science theory and methods applied to mental health in South Asia. A UCL/BALM ${ }^{3}$ research unit was also established in 2008 employing local researchers to conduct joint studies into mental health and its related stigma in the Indian context. Following a brief history and outline of the collaboration, this paper discusses specific challenges: institutional issues and local economics; history and power dynamics; teaching versus training; working within mixed disciplinary and "cultural" domains both in the UK and India; and the spatial and temporal challenges of supervising research across continents. The paper concludes by reflecting on the contribution this collaboration has made to knowledge flow, examining localised and culturally specific understandings of pedagogy. These insights offer potential for similar international organisations seeking to establish inclusive and effective partnerships between frequently disparate contexts.

\section{Introduction}

Teaching in a global setting provides a new focus for anthropologists within the continuing construction and development of education establishments and departments abroad (Costello and Zumla 2000; Caruana and Spurling 2006). There is no template to guide the practical negotiation of complex differences in teaching styles, 
pedagogical understandings and expectations, as well as working across large geographical distances. There are also historical resonances that are especially pertinent for former colonies such as India that have to be recognised and responded to, when developing new teaching projects (Ofori-Adjei and Gyapong 2006; Subedi and Daza 2008). Critiques of teaching in post-colonial settings focus not only on the issue of language, but also the challenges of inhabiting multiple worlds and identities that require teachers, as well as learners to address what knowledge they acquire and how (Bahri 1997; Gorski 2008; Jadhav and Jain 2008; Nandy 1983; Sen 2006; Spivak 1993, 1994). In our collaboration this was a constant and ongoing process whereby structure and content were debated alongside use of terminology, its meaning and the exploration of our individual values and philosophies that arose from our varied cultural backgrounds and identities (explored below). Whilst this paper does not provide a detailed impact assessment, feedback from participants has been included to illuminate the subject matter where appropriate.

Terminology was examined not only to avoid misunderstanding, but also to define meaning within its specific context and there were occasions when "translation" was required; especially how linguistic meanings and concepts were used and understood in both settings - India and the UK. Whilst the term "psychosocial" is commonly used throughout India, "anthropology" still remains (at least outside academic institutions) associated with its historical roots as a theory-driven discipline without obvious application to local mental health problems. In India, an important component of learning and acquiring knowledge, including research, is that it has a clear practical application. This highlighted an initial disciplinary division between our two models; University College London's (UCL) focus on a model that promotes teaching theory, and The Banyan's need to advance the use of applied methods within a specific local context. This resulted, after acknowledgement of the needs of the setting, in a shift by UCL away from an exclusive focus on theory, to a more balanced mix of both theory and applied intervention.

The number of institutional establishments and campuses set up in the international market of education and research is expanding (Hellsten and Reid 2010). Anthropology is well situated to provide input into the development not only of academic curricula but also into the specifics of work in these new globalised academic/teaching environments. It offers a useful perspective in understanding the local challenges involved in teaching not only across disciplines but also within different cultural domains. With its emphasis on cultural specificity, this paper identifies the challenges faced in working across such cultural boundaries from the perspective of our joint collaboration.

\section{The Partnership}

Since 2006, UCL and The Banyan have been collaborating on the development of teaching courses in social science theory and methods, and research in relation to mental health in India. UCL as a high profile university based in London, and The Banyan with her sister organisation, BALM, an influential NGO that supports and advocates on behalf of the mentally ill, cannot necessarily be thought of as equal partners through obvious discrepancies in size, economics and objectives. And yet, through discussions and negotiations held between individuals within each establishment, a partnership was formed that currently conducts regular teaching courses and research.

The initiative began following informal discussions between an Indian trained British based psychiatrist with a $\mathrm{PhD}$ in medical anthropology, and one of the co-founders of The Banyan. Both had shared a desire to bring an alternative and more inclusive engagement between the social sciences and the NGO sector in the development of improved psychiatric theory and practice in India, which still remains rooted in western rather than local texts. There was both a theoretical and clinical gap in how mental health professionals were being trained that was not fulfilled by either mainstream psychiatric training or, for that matter, cultural psychiatry (Littlewood 1990). These courses were aimed at testing how culture and mental health could be better incorporated into a more locally based, culturally sensitive curriculum, in India. These discussions led to a request to the Vice-Provost at UCL for support and funding. Seed funding was granted by the Office for International Affairs, as part of UCL's "Grand Challenges" which called for an inter-disciplinary approach to "Global Health and Citizenship, Intercultural Interactions, and Health and Human Well Being." Funding has also been obtained through BALM's access to government funding, as well as supporters and philanthropists in India, including from the business, media sectors and regular fund-raising events. In 2007, a memorandum of understanding (MoU) was signed between UCL and BALM to continue both its teaching and research initiatives. The MoU was renewed for a further 
period of five years in 2011 with a view to develop joint post-graduate teaching and research programmes, and also involve a prominent Indian social science university ${ }^{4}$.

For UCL, the opportunities to develop local links and to advance social theory and method within the field of mental health in India were inviting. The expectation was that students and researchers would conduct applied research, and also learn about the organisation and approach of this locally based NGO. More significantly, this activity was in keeping with UCL's stated vision of global citizenship and the internationalisation of shared curricula. ${ }^{5}$

For BALM, founded in 2007 as a training, research and advocacy organisation, the aim was to increase stakeholder participation in the mental health sector through the recruitment, training and nurturing of workers who were trained in The Banyan's specific model of care, as well as dissemination of the model both regionally and across India. Added to this was the desire to collaborate in this new endeavour to advance knowledge through an exchange of ideas, skills and experience. The unified aim was to develop and enhance this relationship, which would be mutually beneficial in terms of the aims and objectives that whilst unique were nonetheless also complementary.

As BALM was established with the specific aim of training practitioners in The Banyan's model and to conduct research into various aspects of that model of mental health in the Indian context, the research unit subsequently brought a further collaboration between the two organisations, through funded opportunities from UCL's Global Health Initiative. Two studies have so far been conducted to investigate mental health and stigma in India and involved local researchers who have had an opportunity to gain experience in ethnographic research methods.

Both the teaching and the research unit were established in response to a locally identified need for more grounded teaching in the social sciences and research methods that would address mental illness and marginality in India.

The themes covered by this collaborative activity include an inter-disciplinary approach to mental health dimensions of displacement, disconnection and homelessness, with a focus on the Indian sub-continent. As partners are responsible for delivery of the courses, the aim is also to generate more localised texts and culturally relevant models of practice for mental health practitioners in India - a field that continues to use western-based psychiatric models and texts to inform both theory and service delivery (Jain and Jadhav 2009).

The broad aims of the collaboration are as follows:

- To extend knowledge and build on existing theories that will generate a more inclusive approach to the study and treatment of mental illness in South Asia.

- To develop cross-continent, multidisciplinary collaborations in order to create a dynamic force for change that is inclusive, challenging and vital to the greater understanding of what constitutes "global mental health": including ideas and experiences of suffering and well-being.

- To offer an integrated, intellectual space to address these issues through its research and teaching programme, aided by an experienced faculty that include a multi-disciplinary team of senior clinicians with social science training, and front line staff from the NGO sector.

The UCL team, comprising both mixed primary disciplines and mixed ethnicity and gender, are to some extent more familiar within the multi-cultural context of British academia and National Health Service employment. In total, there are six members of the team: four males and two females of South Asian, British and Nigerian ethnic origins. All academics have clinical qualifications: these are represented by three psychiatrists (all male, including one child psychiatrist); a male social worker; female occupational therapist; and a female intercultural psychotherapist. All of them are trained medical anthropologists/sociologists and each member has conducted either teaching and/or long-term anthropological fieldwork within the region.

The Banyan and BALM team comprises a more dynamic membership, but at its core are the co-founder of The Banyan who is also a psychiatric social worker; the director - who is a researcher and psychologist; all are female and of Indian origin, apart from a resident anthropologist of Dutch origin. There is also a large and changing group of local, trained NGO workers and there is less regular input from a sociologist, an economist, a medical doctor and a psychologist who have also been involved at various times. Also, there are closely involved members of the corporate and development sector who have been instrumental in supporting the grounding of 
The Banyan and continue to have a key role in its financial management and future development planning, all based in India.

Within this mixed membership (for example, in terms of ethnic background, gender and discipline), cultural, disciplinary, social and gender factors emerged and contributed to the ethos of both teams. For example, the UCL team director, from a Dalit caste background has raised contentious issues in relation to teaching caste as a topic on the courses; the mix of disciplinary backgrounds has sometimes lead to hierarchical positioning in terms of roles, both within and across both teams; the ongoing dominance of a bio-medical training has often gone unchallenged by either team; and to a lesser degree, race, gender and religion have all been raised and discussed. Open acknowledgement of these complex issues has been critical in building trust as part of the ongoing development of the partnership, (including designing subsequent teaching courses), if not necessarily their easy resolution. Tensions in relation to social, ethnic, gender and professional identities exist within and across each team, as in any institutional setting. Efforts are made to openly discuss these as a process, not only in generating more effective working relationships, but also because they are key themes within much of the course content.

What both teams share however, is their often marginal status within their own institutional and social settings. This has influenced the identification of themes and methods taught on the courses, which focus on marginality in mental health. For example, each course begins with an exercise that encourages the sharing of some more personal information by each member, led by the teaching team, that relates to cultural, social, and health care interests linked to the theme of the course and how this relates to marginalisation, both within institutional settings and through personal life experiences. Participants reported that this approach helped break down barriers and encouraged more openness and was considered beneficial considering the short time scale of each course.

The partnership's future lies in developing and refining the collaborative relationship in order to deliver informed, creative and ethically based, applied research that will generate texts and locally relevant service delivery models for the sub-continent.

\section{Teaching: Adapting Pedagogical Expectations}

The aims of teaching short courses sited at The Banyan, was two-fold and depended on each partner's overall objectives, namely from UCL's point of view; to encourage a more anthropological or social science perspective on mental health in India (Mishra 2007). The Banyan's objective was to both train (through BALM), and inform participants of their approach and model which could then be disseminated more broadly. They also aimed to provide an integrated approach to mental health care in India that is embedded within a development paradigm. As mentioned in the introduction, following initial recognition of differences in style and emphasis, overall the objectives were complementary. Initially, sessions were allotted in line with each partner's specific aims teaching versus training. UCL focused initially on teaching, including social science theories and The Banyan provided the applied component.

Therefore utilising the respective strengths of each team meant to some degree, dividing up roles and methods rather than a full "intermixing" as had initially been anticipated. BALM primarily trains workers in field-based methods and institutional health care practice as well as advocacy skills, whereas UCL, as stated, primarily teaches theory and encourages exploration through ideas rather than professional training. Bringing the two together required an acceptance of both our strengths and limitations, so that training and teaching became complementary and not divisive, and meant that "teaching" (seen as theory grounded), was generally lead by UCL and training (seen as practice grounded) was lead by BALM. Such "teaching" and "training" were complementary components and approaches whose combination was one of the core strengths of the courses and the collaboration as a whole.

Participant's feedback made it clear that the application of learning was a key element that was wanted on the courses. This meant a shift away from a purely theory-based learning to an integration of theory and practicebased learning through, for example, clinical case material. To avoid an overly classroom-based style, some adaptation was necessary. This meant not only using a lecture format but also, as stated previously, discussions, group work, showing films, and presentation of case studies was required on behalf of participants and teachers, as interactive sessions were introduced. Apart from this, feedback from participants also made it clear that in order to fulfil obligations to employers and funders who had supported their attendance on the course, a clear 
goal/practical oriented outcome was required that could be applied to their workplace setting. The training approach was a better "fit" for demonstrating this.

This has since become the mainstay of our approach but, as stated previously, it is also the case that some reformulation has taken place by both parties in the collaboration. For example, UCL now uses more applied teaching techniques and The Banyan shares its research knowledge as part of the courses. There have also been sessions which have been jointly focused around shared interests: for example, a panel discussion involving a client of The Banyan and her family, a local healer, an NGO worker from The Banyan and cultural psychiatrists. During this session, both theoretical and applied issues were jointly raised and discussed.

\section{The Short Courses}

A series of short courses in the social sciences with a focus on mental health has been held yearly since 2006. The course runs for three days and is organised around specific topics, as follows:

- Basic Introduction to Cultural Psychiatry

- Mental Health and Marginality

- Encounters between Psychiatry and Medical Anthropology

- Recent Advances in Cultural Psychiatry for South Asia

- Re-thinking Psycho-social Interventions in South Asia

- Possession States in South Asia: Theoretical and Clinical Dimensions

- Distress and the Body: Anthropological and Clinical Dimensions

In terms of learning outcomes, the course objectives have included:

- gaining an overview of social science theory and method in South Asia;

- $\quad$ learning key concepts from medical anthropology;

- integrating social science theory with clinical practice;

- applying cultural psychiatry in practice; learning to critique through social science; methods existing theories and practices of western psychiatry;

- determining the relevance of the "folk" sector in South Asia;

- exploring of working with 'healers' and other non-traditional practitioners;

- examining local "idioms of distress;"

- $\quad$ theorizing and critiquing categories of race, caste, gender and its relationship to stigma;

- $\quad$ exploring issues concerning trauma and somatisation from a South Asian perspective.

These courses are aimed at students, researchers and clinicians working in South Asia, as well as external participants with an anthropological interest in the region. So far, there have been 170 participants over the six courses; mainly front-line clinicians from both the public and private sectors in India, and social science researchers including: local NGO workers, psychiatrists, psychologists, social workers, occupational therapists, ayurvedic and homeopathic practitioners, and medical anthropologists as well as teachers. Countries represented include: India, Sri Lanka, Pakistan, UK, the Netherlands, the US, Canada, Italy, Norway and Germany.

Planning the courses, with the separation of members across not only international but also national boundaries in the case of the UK, takes time. Planning for the course starts a year before it takes place and involves multiple emails, phone conversations and discussions negotiating the timetable and tasks, which increase as the course nears. As time has gone on, the majority of the work in planning has now been done in relation to formulating roles: who does what.

Prior to the start of the course, a brochure is prepared outlining course information, timetable of each days content and sent out for each participant in attendance, including the extensive reading list. In addition, 
participants are sent a structured form for submitting case studies. The emphasis is on submitting cases that participants find either clinically challenging or instances where western texts are ineffective in addressing both conceptualisation and management of the presenting problems.

The reading list, selected and prepared by UCL who have better access to resources, incorporates readings from a wide range of literature from medical anthropology, social anthropology, clinical/psychological anthropology, and cultural psychiatry that is relevant to South Asia. Importantly, the UCL team make an attempt to balance the readings with local as well as western or international texts where possible. Finding suitable material that appeals to an audience that is diverse in terms of skills and experiences, is a challenge and therefore both basic and more advanced literature is included. When preparing reading lists, feedback has also been considered, which pointed to the reading list being overwhelming when first received but then it was seen as valuable for post-course reflection. It has also highlighted the lack of locally relevant texts and access issues, whereby articles are not easily available, especially in the topics under discussion on the courses, and for students on low incomes.

Therefore, creating the reading list has provided a useful resource for participants related to the themes of each course and for the partners, can assist with any potential publications in the future. In the student selection process for each course, one of the criteria states that participants must have some familiarity with the basic literature or at least have come prepared, having studied relevant readings; consequently the reading list is posted out as early as possible. Submission of local clinical cases are also requested, for discussion and reformulation through anthropological theory applied to an Indian context. Advertising begins at least six months in advance across the print and web media, and costing is according to local and international rates.

\section{Teaching Methods}

The courses use a mixed methods approach, which utilises a variety of styles and is aimed at ensuring that participants are provided with varied learning experiences through techniques such as: lectures, seminars, case studies, group work, films, role play, field visits, debates and panel discussions. Peer learning is also encouraged and participants bring case studies to be worked on over the duration of the course, taken from their particular work settings. Participants reported finding the mix of theory, practical group exercises, case-based discussion, and film an effective method of learning.

Interactive teaching methods were key to the learning process and enabled misunderstandings to be clarified and discussed; this has evolved as it was realised that not only are learning styles predominantly "by rote" for many participants, but also time is limited. Considering the mixed nature of the group, early interaction encourages participants to settle quickly within a conducive atmosphere. Participants valued the ethos of reflective learning that also included group discussion and feedback sessions at the end of each days teaching. Additionally, a summary of each days teaching content was requested by participants and subsequently, and it has received favourable feedback.

The introduction session mentioned above, was not only as an ice-breaker, but also allowed a shift in pedagogical thinking and method - one that was commented on by some participants as contrasting with what was seen as the more "traditional" teaching methods in many institutions in India. Generally, our teaching has involved a mixed media approach; for example, sharing clinical case vignettes or fieldwork experiences has expanded the perspectives of both types of participant and provided effective learning opportunities that also reflect a desire to bring the two "worlds" closer together.

It has been important having a mixed audience where participants (and teachers) learn from each other not only subject matter, but also - approaches. Participants come from varied social science and clinical backgrounds, as well as different stages in their education and training. This necessitates a more imaginative use of teaching methods reflecting their different experiences and backgrounds. The approaches used are under constant review based on feedback at the end of each course, as well as the daily feedback.

Interestingly, these differences between a clinical and social science perspective has mirrored the differences in approach and attitudes more broadly within the fields of medicine and the social sciences in the country. Indeed, one of the key achievements of the courses has been the development of a dialogue between the language of social science and that of mental health in India - toward a cross-fertilisation of ideas, methods and skills. By ensuring that each session revolved around clinical case based discussions and 
the subsequent introduction of anthropological concepts and methods, the participants were able to link the specialised languages of psychiatry and medical anthropology with more immediate practical dilemmas in the clinic setting. Several participants commented on the value of learning how to connect local, academic and clinical perspectives, within their own contexts.

Each course is followed by a structured post-course debrief meeting of the teaching faculty, where planning for the subsequent years' course takes place. This also includes a re-examination of each other's expectations in terms of the collaboration; feedback from course participants ${ }^{6}$; and ways of improving and developing the collaboration more generally.

\section{Research}

The collaboration is concomitantly driven by a desire to extend research capacities for academics and their students locally and from UCL, through access to field sites and informants and to advance relevant research. This provides a unique opportunity not only to build local capacities but also to exchange programmes of training and education in the social sciences in relation to mental health. There are two main research objectives therefore: firstly, to enhance the capacity of local health researchers interested in the social sciences, through both training and joint research initiatives. This is aimed at developing and promoting a more culturally appropriate methodology. Secondly, to conduct new research that is of interest to both partners. Two pilot studies have been carried out so far that provided local investigators with supervised experience in conducting small research projects within the newly established research unit.

Supervision of local research assistants was an especially rewarding and important learning opportunity, for both new researchers and supervisors. To date, two collaborative studies have been undertaken within the UCL/BALM research unit: the first one, looking at stigma towards mental illness among the population in South India; and, the second one, a small ethnography of a community living project run by The Banyan (Derges et al. 2012). The latter study involved two stages and two different research assistants; both had had medical training and were interested in gaining experience of social science/anthropology methods of interviewing and ethnography, although neither had conducted similar research before.

The first fieldworker-researcher was employed for a period of five months and supervised at a distance via phone and email, before a final meeting towards the end of her study. The second research assistant was introduced to the field site and some basic techniques of ethnographic participant-observation over a period of a week by one of the UCL members prior to the project beginning. Following on from this initial introduction, contact with the research site was maintained via Skype and email. There were key experiential differences between the research assistants and the supervisor: phone supervision alone was understandably less beneficial for the first research assistant and although good data was obtained, there was less opportunity to discuss methods and issues that arose as they happened. Data obtained from the second study was qualitatively rich, mostly due to the ethnographic methods employed and it was easier to monitor progress having had this initial face-to-face introduction, which built trust. Regular skype calls further helped develop the supervisor/researcher relationship. This interpersonal knowledge was especially important as part of the ethnography in a mental health community project. It was also highly demanding due to the complex nature of the setting, including the parttime residency among individuals suffering severe mental health problems. The supervisor stated that supervision at a distance was also a challenge in terms of technology, time limitations, distance, and lack of regular opportunities for on-site interaction.

\section{The Language of Teaching}

Throughout, clarification of terms was required time in order to arrive at shared understanding. Although this slowed things down, it was necessary in order to reach a similar understanding. The majority of UCL faculty have conducted fieldwork in India and are fluent in one of the several languages spoken in the country, which helped considerably in unpacking these issues 7 . The Banyan members contributed and ensured more in depth knowledge and experience in this process. 
For example, The Banyan and the UCL partners did not have shared meaning about the concept of "culture;" the UCL team had greater, although not complete, familiarity with the terms' usage in anthropology - at least with regard to the debates about utility. Initially, there was a wrong assumption that the notion of "culture" and its relevance to teaching were equally understood by all members of the collaboration. The Banyan did not share this preoccupation with defining "culture" and teaching "about culture" unlike the UCL staff, for whom "culture" was the mainstay of teaching in anthropology. After much discussion, it was ultimately recognised that this different understanding of "culture" was about different interpretation in relation to the salience of "culture" rather than its definition. This reduced its problematic role as increasing the tension between teaching and training. The local Indian equivalent of the term "culture" was in fact reframed as "social" and encompassed more operational terms of "culture" such as poverty, rights of the mentally unwell, discrimination, and lack of access to health care.

\section{The Challenges}

The partnership has gone through various stages of development and learning in relation to its different agendas: financing; the need to bring in participants for the courses; providing evidence of success for future funding; promoting an understanding of what is meant by "culture;" developing methodologies in "teaching" versus "training;" and, resolving the spatial-temporal challenges of supervision.

These key challenges have been addressed alongside progress made in planning and implementing the courses and research.

In sum, the success of the courses hinged on understanding not only cross-disciplinary methods in teaching, but also cultural and geographical boundaries with their complex nuances of language and terms that had to be continuously negotiated, responded to and re-formulated by all partners. Understanding these issues proved to be an important learning opportunity for all and could not have been otherwise anticipated. For both sets of collaborators who are positioned at the margins of their respective fields, there have been challenges. For example, the position of cultural psychiatry in relation to the broader more dominant field of biological psychiatry, and the position of The Banyan with regard to the mental health sector in India that remains dominated by medicine, affects the progress of the collaboration. Planning and development are a constant source of debate in the course of seeking ongoing funding.

\section{The Partnership Dynamic}

Historically, India as a former British colony has been influenced by a Raj-style pedagogy ${ }^{8}$. Despite a residual teaching style that remains close to its colonial roots (rote learning within a non-interactive classroom environment), progress has been made in attendance and literacy rates. Now, India competes internationally through its many world-class universities and educational/training institutes and colleges. Alongside this there is a newer influx of interested international academic institutions and researchers, eager to benefit from the "New India" and who, it is often feared will take knowledge away and use it to further their own academic careers, without benefit to local institutions (Costello and Zumla 2000). A more recent critique suggests that the "internationalisation" of education is nothing new (Pietsch 2012; Cousin 2011). Pietsch (2012) refers to the similarities between the current and the imperial system, where accreditation of degrees obtained externally was biased, as they were not universally accorded the same value as those obtained from British based universities. This meant that graduates with the same degrees obtained from universities outside Britain, faced difficulties having their qualifications recognised or accepted when applying for jobs in the UK. In the current climate of high fees and global campuses, there is an increasing risk of a widening the gap between those able to afford a western education, and the continuing domination of education systems that disseminate western theories, methodologies, nosologies and language.

International NGOs compete for presence in India today and this influences existing partnerships. For example, UCL is not the only university with which The Banyan has an MoU. It can be said that from the perspective of 
NGOs such as The Banyan, this kind of competition is important within a context of funding shortages. However, BALM's primary aim is not income generation; it is to increase human resources in the mental health sector in India. The main input from these institutions therefore, is to provide greater academic rigour.

Producing and sharing new knowledge is an important aim for both partners and has been a point of negotiation in relation to ownership of data from both the short courses and the research outputs. New knowledge has been generated and shared through writing, planning new research, and developing courses. It has required trust, sensitivity and ongoing discussion of these points. For example, discussion and negotiation as to what happens to material generated through the courses or research studies, including who has access and for what purpose, has been an ongoing discussion. These issues emerged slowly through informal discussions in relation to professional experience, qualifications and possession of local knowledge. Local knowledge in particular, was understandably the most contested area - and the most prized; the belief being that The Banyan/BALM members held local knowledge and expertise, while the clinician academics held the theoretical knowledge current in the UK at present. Whilst this was partially true, it was mostly however, a "belief", whereas in reality all partners had contributions to make in theorizing, and everyone contributed with their local expertise. Trust has been built gradually (distance being a constraining force) through both informal and formal means. Informal social gatherings during visits are an important way of getting to know each other better in a non-teaching context. More formally, it was critical to allow time for the development of the partnership, especially in understanding attitudes to ethical practice, and approaches to mental health practices. Each visit contains a scheduled, recorded meeting to discuss the collaboration and its future, which is then followed up over the ensuing year. Visits by both teams to their respective work locations have helped develop an understanding of each institution's ethos, as well as advantages and constraints. The Banyan have been able to attend three meetings in person in London, and we maintained an ongoing contact through Skype, telephone, and email exchanges. This has facilitated the sharing of experience and expertise with regards to the courses and research. This is an area of ongoing cross-fertilisation, as ideas and understandings emerge that need to be understood and exchanged; particularly in relation to local terminologies (including defining "culture"), mental health theories, expertise and knowledge.

There is another dynamic that relates to different concepts of "time." UCL operates within a system defined through academic structures, which divides time into portions. A Western post-capitalist economy and culture also predominantly perceive speed as equalling efficiency; therefore scheduling and operating to strict deadlines is more common. An NGO has very different constraints based in part on economic factors ensuring its ongoing existence. This means, priorities relating to the attainment of funding have to be emphasized, including publicity and dissemination of the work, for example. There is also perhaps less emphasis on schedules and timescales in the fulfilment of objectives. It also has to be remembered that for both UCL and The Banyan/BALM, the partnership is only one aspect of their work; both teams also work clinically and in teaching and research in their respective organisations.

For Higher Education (HE) institutes in countries such as the UK, finding suitable placements for students with regards to finances and experience is a priority when considering options for studying abroad. However, there is nervousness in fully committing to teaching ventures, especially those outside an established academic institute. Due to potentially increased financial instability, NGOs may be considered high risk when it comes to investment by academic institutions.

\section{Economics and the Challenges of Funding}

Economics was perhaps the most complex and continuous area of negotiation that highlighted the differences between both institutions. On the surface, it would seem likely that a UK institution such as UCL would be able to fund the courses with relative ease. Constraints on institutional and higher education funding and fewer grant giving bodies has meant $a d$ hoc and piecemeal financing of the courses. This is not due to dissatisfaction or to a lack of interest but mainly - to less money in the pot. Priority is given to evidence of the success. What are they bringing in terms of financial and academic rewards? Are Research Excellence Framework (REF) points going to result from this work, in relation to the assessment of its impact as a research output, rather than a teaching output? Initiating collaborations of this kind with international partners, especially where there are different and unequal financial constraints, takes time. In the early stages, economic risk is required without the necessary reward of an immediate research output, as collaboration needs time to fertilise and produce work that will produce a worthwhile financial benefit for the home institution. 
Similarly, The Banyan and BALM also have considerable financial constraints and rely on the benevolence of philanthropists and supporters. There is now government backing through grants and The Banyan is now part of a government mental health policy group. However, in terms of a more radical creative re-thinking of mental health care in the country, NGOs such as The Banyan - let alone research and training departments like BALM, struggle to develop when there is little security in its future. UCL also finds it challenging year on year, to find financial backing to support the ongoing collaboration. The effect of this challenge has been to delay longer term planning and commitment to future research development and the courses themselves are under constant threat of being withdrawn. A further economic consequence of this relates to participant numbers. This means balancing the desire for as many partners as possible to boost the financial remunerations, against weighing the need for courses to be small enough, so that everyone contributes and benefits. Local participants regularly struggle to obtain funding and international participants must find airfares and accommodation, which adds to the already higher fees - and many of these participants are students on limited budgets.

\section{Conclusion}

The collaboration between UCL and The Banyan began on the basis of shared teaching on short courses, aimed at a local NGO and health workers interested in the social sciences and in mental health practice development. The collaboration arose out of concerns that local pedagogies in India were still predominantly based on western texts and not inclusive of local culture. "Cultural psychiatry" is able to fill this gap with locally based theories and nosologies. Courses were also predicated on a desire to challenge recent notions of a Global Mental Health movement by providing a contextualised perspective on issues that were of more specific local relevance (McCoy et al. 2008). Later came the goal to develop a research unit that would support both local and UCL research initiatives. This has developed with an increasingly mixed audience and, whilst the original intention still remains, the courses have progressed to the point of a full programme module.

The process of connecting UCL academics with a grass roots organisation in a low-income country has meant acquiring a double consciousness or an intellectual diplopia. In doing so, these insights have been deployed within the university setting through internationalising the curricula and teaching. Recognising that cultural issues impact upon staff-student relationships and learning outcomes has led to UCL establishing a unique Cultural Consultation Service (CCS) for Staff and Students?.

For The Banyan and BALM team, the collaboration has provided an alternative theoretical perspective that is more accommodating of the locally specific setting. The exchange of ideas, knowledge and experience has supported mental health care development in the country. This exploration is continuous and incomplete as yet, as the ideas and practices are being digested, expanded and incorporated. But the collaboration has indicated a path that is more inclusive of the very particular problems faced by sufferers of mental illness in India and it has only been beneficial to those involved in their care.

The current challenge is how to take the collaboration forward. Overall, there has been much re-thinking and adjustment in terms of identifying appropriate pedagogies, understanding of power dynamics, negotiations around financing and economics, and adjustment of expectations and attitudes. Lengthy discussions have taken place over the years that have developed not only teaching, but also research activities that involve planning the future direction of the collaboration. There is still a long way to go, and the current challenges address mainly this future direction. There is a willingness to continue on the part of all collaborators, but there remain severe constraints that are mostly institutional and economic. Both involve certain risks. Some of the main risks pertain to the development of trust and others result from the uncertain funding - there is a point after which more sustainable financial support will be indispensable, and, in the current climate of cutbacks and institutional nervousness in relation to "riskier" ventures, such financial sustainability appears increasingly remote.

\section{Notes:}

1 "Clinician Anthropologist" is defined here as anthropologists with clinical training in the field of medicine or health-related professions.

${ }_{2}^{2}$ Mental Health Sciences Unit, University College London (UCL), and The Banyan, a Indian NGO in Chennai that supports individuals with mental health problems, including the homeless. 
${ }^{3}$ BALM: Banyan Academy of Leadership in Mental Health.

${ }^{4}$ http://www.thehindu.com/health/medicine-and-research/article1603097.ece. (Retrieved 16 March 2012)

${ }^{5}$ http://www.ucl.ac.uk/global (Retrieved 16 March 2012).

${ }^{6}$ A full impact assessment is currently in progress.

${ }^{7}$ Language is a topic that has been raised by participants on the short courses: the need to develop local terminologies that are inclusive of local nosologies (this refers to local indigenous classifications of illness, disorders and diseases)

8 A "raj-style pedagogy," refers to the teacher student relationship in India, which is rooted in a skewed interpretation of pre-colonial Indian cultural pedagogy and re-enforced by alienated teachers (Gurus) of Indian psychiatry who in turn, have successfully internalised European psychiatric philosophy. As a consequence, local psychiatry including its theory and practice is culturally invalid. An oppressive and stagnant dynamic between contemporary Indian teachers and their students, further perpetuates the alienated connection between doctors and their patients; urban health planners and their rural recipients; and between public mental health service providers and users.

9 (<http://www.ucl.ac.uk/ccs>). (Retrieved 16 March 2012).

\section{Source material:}

http://www.thehindu.com/health/medicine-and-research/article1603097.ece. (Retrieved 19 January 2012). http://www.ucl.ac.uk/global. (Retrieved 21 March 2012). http://www.balm.in/doc/UCLBALMResearchUnit.pdf (Retrieved 15 March 2012). http://balm.in/home.html (Retrieved 15 March 2012). http://www.ucl.ac.uk/ccs (Retrieved 12 December 2012)

\section{References:}

Bahri, D (1997), 'Marginally Off-Center: Postcolonialism in the Teaching Machine,' College English, 59 (3): $277-$ 298.

Caruana, V. and Spurling, N. (2007), The Internationalisation of UK Higher Education: A Review of Selected Material,

York: Higher Education Academy, http://www.heacademy.ac.uk/resources/detail/the_internationalsiation_of_he (Retrieved 11 December 2012).

Costello, A., Zumla, A. (2000), 'Moving to Research Partnerships in Developing Countries,' BMJ, 321: 827-829.

Cousin, G. (2011), 'Rethinking the Concept of "Western",' Higher Education Research and Development, 30 (5): $585-$ 594.

Derges, J., Jadhav, S., Narayan, L., Gopikumar, V., Littlewood, R. (2012), 'Interdisciplinary Research Project on Stigma of Homeless Women with Mental Illness in South India,' poster presentation, UCL Grand Challenge of Global Health (GCGH): Small Grants for 2010.

Gopikumar, V., Negi, E., and Dijkxhoorn, M. (2012), 'The Role of Non-Governmental Organisations in Community Mental Health Care,' in B.S. Chavan, N. Gupta, P. Arun, A. Sidana, and S. Jadhav (eds) Textbook of Community Mental Health in India. Jaypee: New Dehli India, pp. 148-158.

Gorski, P.C. (2008), 'Good Intentions Are Not Enough: A Decolonizing Intercultural Education,' Intercultural Education, 19 (6): 515-525.

Hellsten, M., and Reid, A. (eds.) (2010), Researching International Pedagogies: Sustainable Practice for Teaching and Learning in Higher Education. Dordrecht, The Netherlands: Springer Science and Business Media B.V.

Jain, S. and Jadhav, S. (2008), Cultural History of Community Psychiatry in India,' International Journal of Health Services, 38 (3): 561-584.

Jadhav, S. (2012), 'Cultures of Teaching and Global Well-being,' seminar paper at Global Citizenship as a Graduate Attribute: an ESRC Seminar Series, http://www.wlv.ac.uk/globalcitizen (Retrieved 24th March 2012).

Jain, S. and Jadhav, S. (2009), 'Pills That Swallow Policy: Clinical Ethnography of a Community Mental Health Programme in India,' Transcultural Psychiatry, 46 (1): 60-85.

Littlewood, R. (1990), 'From Categories to Context: A Decade of the New Cross-cultural Psychiatry,' The British Journal of Psychiatry, 156: 308-327.

McCoy, D., Mwangambo, C. and Costello, A. (2008), 'Academic Partnerships between Rich and Poor Countries,' Lancet, 371: 1055-7.

Mishra, A. (2007) 'Teaching Medical Anthropology in India' Anthropology News. March 2007, pp. 27. 
MoU to facilitate cross-cultural research (2011). BALM, TISS and University College London, The Hindu. Chennai edition, 6 April 2011, http://www.thehindu.com/health/medicine-andresearch/article1603097.ece (Retrieved 8 April 2011).

Nandy, Ashis (1983), The Intimate Enemy: Loss and Recovery of Self Under Colonialism. Delhi: Oxford University Press.

Ofori-Adjei, D., and Gyapong, J. O. (2006), 'A Developing Country Perspective on International ResearchPartnerships on Health,' http://www.nuffic.nl/home/newsevents/docs/events/kotm/abstracts (Retrieved 16 March 2012).

Pietsch, T. (2012), 'Imperial Echoes' Time Higher Education, 8 March 2012, http://www.timeshighereducation.co.uk/419239.article (Retrieved 8 $8^{\text {th }}$ March 2012).

Sen, A. (2006), Identity and Violence: The Illusion of Destiny. Penguin: London.

Spivak, G.C. (1993), Outside the Teaching Machine New York: Routledge.

Spivak, G. C. (1994), 'How to Read a Culturally Different Text,' in F. Barker, P. Hulme, and M. Iversen (eds) Colonial Discourse/Postcolonial Theory, Manchester: Manchester University Press, pp. 126-50.

Subedi, B., Daza S.L (2008), 'The Possibilities of Postcolonial Praxis in Education,' Race, Ethnicity and Education, 11 (1): 1-10. 